\title{
STUDY OF STRUCTURAL PHASE TRANSITION IN MgSe
}

\author{
Ravindra Pandey and Amin Sutjianto \\ Department of Physics, Michigan Technological University ,Houghton, MI 49931
}

\author{
(Received 2 March 1994,accepted for publication 11 May 1994 \\ by A.H.MacDonald)
}

\begin{abstract}
Total energy of $\mathrm{MgSe}$ as a function of unit cell volume has been calculated for the wurtzite, zinc-blende, and rocksalt phases by the periodic Hartree-Fock method. The calculated results suggest the crystallization of $\mathrm{MgSe}$ in the wurtzite phase at ambient conditions. No phase transition from wurtzite to zinc-blende is predicted which is found to be about $0.05 \mathrm{eV}$ higher than wurtzite at equilibrium volume. For the phase transition to the rocksalt phase, the transition pressure comes out to be about $60 \mathrm{GPa}$. Analysis of electronic structure at equilibrium volume shows the minimum band gap to be indirect in the high-pressure phase of $\mathrm{MgSe}$.
\end{abstract}

There has been recent interests in Magnesium Selenide as a cladding layer for $\mathrm{ZnSe}$-based diode lasers and as a key component in large band-gap semiconductor alloys having both $n$ and $p$ type doping ability. It has also been suggested that $\mathrm{MgZnSe}$ alloy lattice can easily be matched with III-V alloy substrates for device applications. [1,2] In these studies, the crystal structure of MgSe was taken to be either zinc-blende with $a=5.45 \AA$ [3] or rocksalt with $a=5.463 \AA$ [4] . However, thin films of MgSe have also been shown to crystallize in the wurtzite phase with lattice constant of $4.145 \AA$ and $c / a$ of 1.622.[5] This ambiguity about the crystallization of MgSe at ambient conditions has led us to study its phase equilibria involving wurtzite, zincblende and rocksalt phases from theoretical standpoint.

Our theoretical approach is based on the $a b$ initio periodic Hartree-Fock (HF) approximation which uses linear combinations of Gaussian orbitals to construct a localized atomic basis from which Bloch functions are constructed by a further linear combination with plane-wave phase factors. These Bloch functions are the basis in which the Fock operator is diagonalized and in terms of which its eigenvectors are expanded.[6] In this work, Gaussian basis

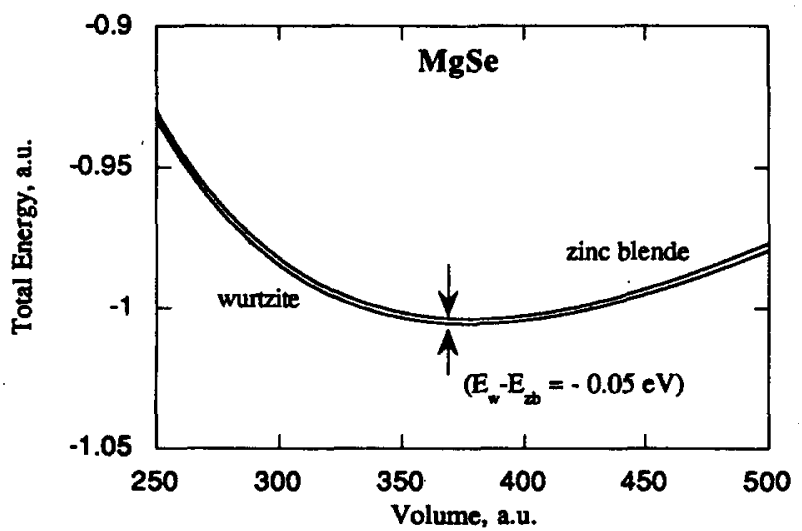

Fig. 1: Total energy (per formula unit) vs. volume per formula unit for the wurtzite and zinc-blende phases of MgSe. sets consisting of two $s p$ shells have been used to describe valence electrons and effective core pseudopotentials for core electrons of $\mathrm{Mg}$ and Se.[7, 8] This periodic pseudopotential HF approach implemented in CRYSTAL 92 program has shown to predict accurate structural properties for III-V and IV-IV semiconductors. [9] For the basis set, the exponents of the innermost shell are obtained by the atomic optimization while the outermost exponents are optimized in the crystalline state yielding the $s p$ exponents of 0.3 and 0.13 for $\mathrm{Mg}$ and $\mathrm{Se}$ respectively. It is to be noted here that we have used the same basis set for the wurtzite, zinc-blende and rocksalt phases and a relatively high precision level for calculations.

We begin with a calculation of total energy as a function of unit cell volume. Both the zinc-blende and rocksalt phases can be characterized by a single structural parameter, the cubic lattice constant $a$ with the primitive unit cell volume of $a^{3 / 4}$. For the hexagonal wurtzite phase, there are three parameters: the basal plane lattice constant $a$, the uniaxial lattice constant $c$ and the internal coordinate $u$ that determines the relative position of the anion and cation sublattice along the $c$-axis. We therefore minimize the total

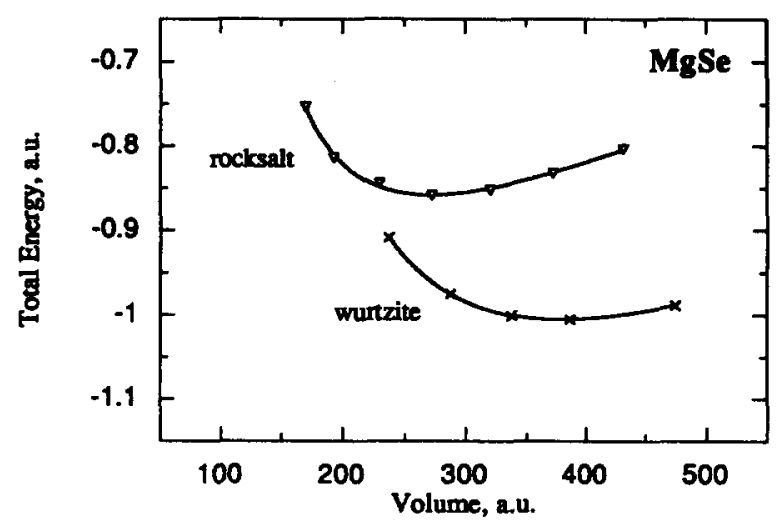

Fig. 2: Total energy (per formula unit) vs. volume per formula unit for the wurtzite and rocksalt phases of MgSe. 


\section{Table I}

Structural properties of the wurtzite, zinc-blende and rocksalt phases of MgSe. All extensive quantities are per formula unit.

\begin{tabular}{clll}
\hline \hline & Wurtzite & Zinc blende & Rocksalt \\
\hline Experiment & & & \\
$a(\AA)$ & $4.145^{\mathrm{a}}$ & $5.89 \mathrm{~b}$ & $5.463 \mathrm{c}$ \\
$c / a$ & 1.622 & --- & -- \\
Present work & & & \\
$a(\AA)$ & 4.319 & 6.072 & 5.434 \\
$c / a$ & 1.602 & --- & --- \\
$u$ & 0.38 & --- & -- \\
$\Delta E d(e V)$ & --- & 0.05 & 5.41 \\
$B_{0}(G P a)$ & 56 & 58 & 68 \\
$B^{\prime}$ & 3.07 & 2.89 & 4.04 \\
\hline
\end{tabular}

aRef. 5

bRef. 2, extrapolation from $\mathrm{ZnMgSe}$ alloy.

CRef. 4

dEnergy difference between wurtzite and zinc-blende / rocksalt phases at equilibrium volume.

energy with respect to $c / a$ and $u$ at fixed volume $\left(3^{1 / 2} a^{2} c / 4\right.$ per formula unit) for each volume we consider. (The details of this optimization procedure are the same as in the reference 10.) The equilibrium structural parameters (total energy, lattice constant, bulk modulus and its pressure derivative) are found by fitting the energy-volume data to the Murnaghan equation [11]

Figs. 1 and 2 show the calculated total energy as a function of the volume (both per formula unit) of wurtzite

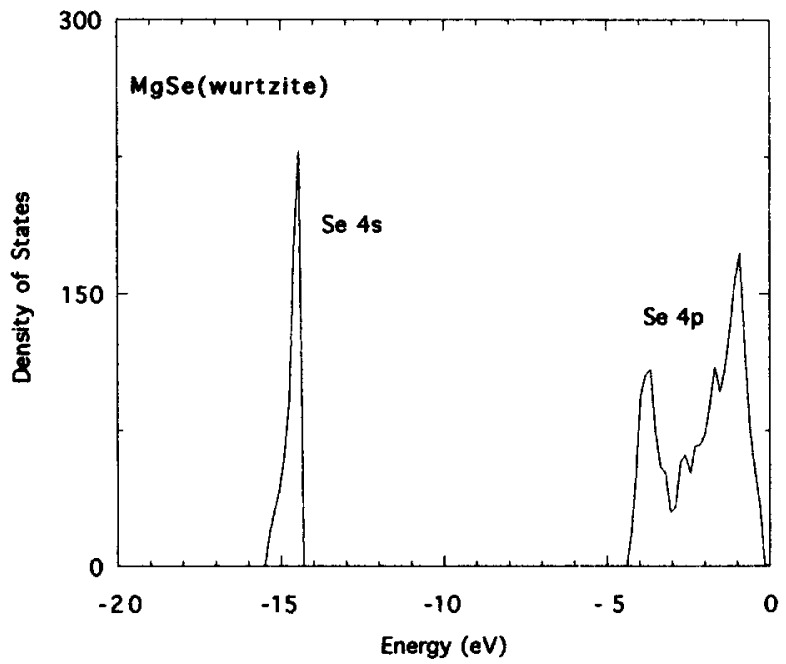

and zinc-blende and wurtzite and rocksalt phases respectively. The equilibrium volume and bulk modulus of the zinc-blende phase are predicted to be similar to those of the wurtzite phase while the total energy is $0.05 \mathrm{eV}$ less, consistent with wurtzite being the ground state. No highpressure phase transition from wurtzite to zinc-blende is predicted. From the common tangent, the phase transition from wurtzite to rocksalt is predicted to occur at about 60 $\mathrm{GPa}$ accompanied by a volume contraction of about $19 \%$.

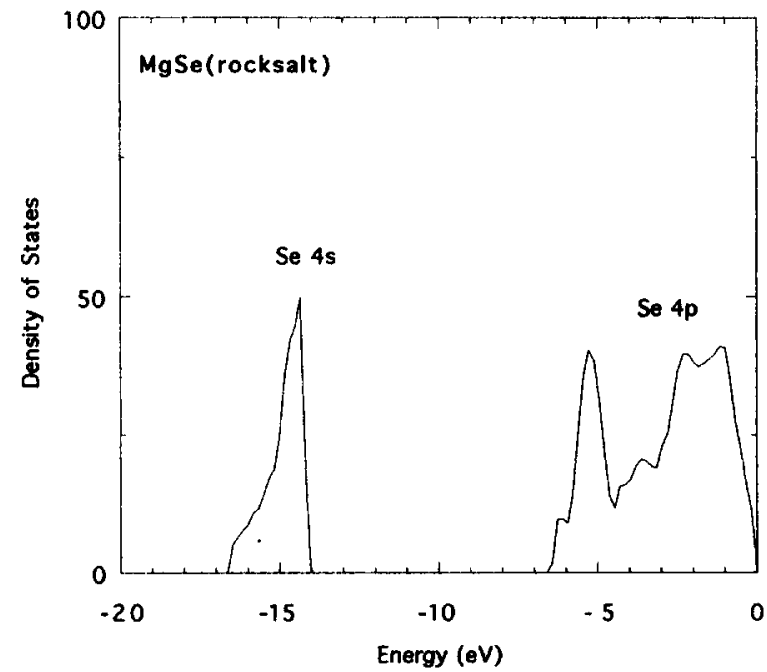

Fig. 3 : Density of States of $\mathrm{MgSe}$ in the wurtzite and rocksalt phases. 
The lattice constant in the zinc-blende phase has been reported to be $5.45 \AA$. [3] However, it comes out to be $5.862 \AA$ when we assume the density of zinc-blende to be the same as of the wurtzite. The extrapolation of the lattice constant from $\mathrm{ZnMgSe}$ alloy yields the value of $5.89 \AA$ [2] whereas Table 1 shows the calculated value of $6.072 \AA$. For the rocksalt phase, the calculated value of $a$ is $5.434 \AA$ in comparison to the reported values of $5.463 \AA$ [4] or 5.449 A. [12]

Next we briefly comment on the electronic structure of MgSe. In all the three phases considered here, the valence-band maximum occurs at $\Gamma$. The calculated band structure is similar to one reported earlier using Hartree-Fock approximation with the mixed basis. For the rocksalt phase, the direct gap at $\Gamma$ was found to be about $9.9 \mathrm{eV}$. The conduction band minimum was at $\mathrm{X}$ with the indirect gap of
$4.1 \mathrm{eV} .[13]$ Note that Hartree-Fock approximation is known to overestimate band gaps relative to optical data. The density of states are shown in Fig. 3. The upper valance bandwidth is 4.1 and $6.3 \mathrm{eV}$ in the wurtzite and rocksalt phases respectively. The larger bandwidth in the (denser) rocksalt phase is the result of more overlap between neighboring atoms.

In summary, we have provided a theoretical basis for understanding the phase equilibrium of $\mathrm{MgSe}$. Our calculations predict the crystallization of $\mathrm{MgSe}$ in the wurtzite phase at ambient conditions. Its transition to the rocksalt phase is predicted about $60 \mathrm{GPa}$. It is hoped that this study will provide a stimulus for experimental investigations which will result in the full exploitation of such an important material for device applications.

\section{REFERENCES}

1. D. J. Chadi, Phys. Rev. Letts., 72, 534 (1994).

2. H. Okuyama, K. Nakano, T. Miyajima and K. Akimoto, J. Cryst. Growth, 117, 139 (1992).

3. I. Broser et al., in Physics of II-VI and I-VII Compounds, edited by O. Madelung, Landolt-Bornstein, Vol 17, Pt. B (Springer, Berlin, 1982).

4. Crystal Data, Vol II : Inorganic Compounds, edited by J. H. Donnay and H. M. Ondik ( U. S. Dept. of Commerce).

5. H. Mittendorf, Z. Physik 183, 113 (1965).

6. C. Pisani, R. Dovesi and C. Roetti, Hartree-Fock $a b$ initio Treatment of Crystalline Systems, (SpringerVerlag, Berlin, 1988); R. Dovesi, V. R. Saunders and C. Roetti, CRYSTAL92 User Documentation, University of Torino and Daresbury Laboratory (1992).
7. B. Silvi, M. Causa, R. Dovesi and C. Roetti, Mol. Phys. 67, 891 (1989).

8. Y. Bouteiller, C. Mijoule, M. Nizam, J. C. Barthelat, J. P. Daudey, M. Pleisser and B. Silvi, Mol. Phys. 65, 295 (1988).

9. M. Causa, R. Dovesi and C. Roetti, Phys. Rev. B 43, 11137 (1991).

10.For details, we refer to the work of J. E. Jaffe and A. C. Hess, Phys. Rev. B 48 (1993) on ZnO.

11.F. D. Murnaghan, Proc.Nat. Acad. Sci. U.S.A. 30, 244 (1944).

12.S. D. Chaturvedi, S. B. Sharma, P. Paliwal and M. Kumar, Phys. Stat. Sol (b) 156, 171 (1989).

13.R. Pandey, P. Lepak and J. Jaffe, Phys. Rev. B 46, 4976 (1992). 